\title{
Theory and practice of ambiguity labelling with a view to interactive disambiguation in text and speech MT
}

\author{
Christian Boitet
}

\author{
GETA, CLIPS, IMAG (UJF \& CNRS), \\ 150 rue de la Chimie, BP 53 \\ 38041 Grenoble Cedex 9, France \\ Christian.Boiteteimag. fr
}

\begin{abstract}
In many contexts, automatic analyzers cannot fully disambiguate a sentence or an utterance reliably, but can produce ambiguous results containing the correct interpretation. It is useful to study vatious properties of these ambiguities in the view of subsequent total or partial interactive disambiguation. We have proposed a technique for labelling ambiguities in texts and in dialogue transcriptions, and experimented it on multilingual data. It has been first necessary to define formally the very notion of ambiguity relative to a representation system, as well as associated concepts such as ambiguity kernel, ambiguity scope, ambiguity occurrence.
\end{abstract}

Keywords: interactive disambiguation, ambiguity labelling, ambiguity occurrence, ambiguity kernel

\section{Introduction}

We are interested in improving the quality of MT systems for monolinguals, where the input can be text or speech, no revision is possible, and the controlled language approach is not usable. In such contexts, the automatic analyzer cannot fully and reliably disambiguate a sentence or an utterance, and the best available heuristics don't select the correct results often enough. Complete or partial interactive disambiguation, following a best possible automatic disambiguation, is an attractive way to raise quality and reliability.

To develop good strategies for interactive disambiguation, it is useful to study vatious properties of the ambiguities unsolvable by state of the art analyzers.

To conduct such studies, it is necessary to gather data, that is, to perform "ambiguity labelling" on texts and transcriptions of spoken dialogues. Our motivations and goals are explained in more detail in the first part. As the usual notion of ambiguity is too vague for our purpose, it is necessary to refine it. This is done in the second part, where we define formally the notion of ambiguity relative to a representation system, as well as associated concepts such as kernel, scope, occurrence and type of ambiguity. In the third part, we propose a format for ambiguity labelling, and illustrate it examples from a transcribed dialogue. This format is independent of the exact kind of output produced by any implemented analyzer, essentially because ambiguities are described with a view to generate human-oriented questions.

We have experimented our technique on various kinds of dialogues and on some texts in several languages. In some cases, analysis results produced by automatic

\author{
Mutsuko Tomokiyo
}

\author{
ATR Interpreting Telecommunications Research Labs \\ 2-2 Hikari-dai, Seika-cho, Soraku-gun \\ Kyoto 619-02, Japan \\ tomokiyodi.tl.atr.co.jp
}

analyzers were available, in others not. It is interesting to compare the intuition of the human labeller with results actually produced: most of the time, differences may be attributed to the fact that available analyzers don't yet match our expectations for "state of the art" analyzers, because they produce spurious, "parasite" ambiguities, and don't yet implement all types of sure linguistic constraints.

\section{Motivations and Goals}

Interactive disambiguation technology must be developed in the context of research towards practical Interpreting Telecommunications systems as well as high-quality multitarget text translation systems. In the case of speech translation, this is because the state of the art is such that a black box approach to spoken language analysis (speech recognition plus linguistic parsing) is likely to give a correct output for no more than 50 to $60 \%$ of the utterances ("Viterbi consistency" [2] $)^{1}$, while users would presumably require an overall success rate of at least $90 \%$ to be able to use such systems at all. However, the same spoken language analyzers may be able to produce sets of outputs containing the correct analysis in about $90 \%$ of the cases ("structural consistency" [2]) ${ }^{2}$. In the remaining cases, the system would be unable to analyze the input, or no output would be correct.

Further extralinguistic and sure disambiguation may be performed (1) by an expert system, if the task is constrained enough; (2) by the users (author or speakers), through interactive disambiguation; and (3) by a (human) expert translator or interpreter, accessible through the network. For example, an expert interpreter "monitoring" several bilingual conversations could solve some ambiguities from his workstation, either because the system decides to ask him first, or

1 According to a study by Cohen \& Oviatt, the combined
success rate (SR) is bigger than the product of the indivi-
dual success rates by about $10 \%$ in the middle range.
Taking S2 = S1 ${ }^{*}$ S $1+(1-\mathrm{S} 1)^{*} \mathrm{~A}$ with $\mathrm{A}=20 \%$, we get:
\begin{tabular}{|c|c|c|c|c|c|c|c|c|}
\hline SR of 1 component (S1) & $40 \%$ & $45 \%$ & $50 \%$ & $55 \%$ & $60 \%$ \\
\hline SR of combination (S2) & $28 \%$ & $31 \%$ & $35 \%$ & $39 \%$ & $44 \%$ \\
\hline S1 & $65 \%$ & $70 \%$ & $75 \%$ & $80 \%$ & $85 \%$ & $90 \%$ & $95 \%$ & $100 \%$ \\
\hline S2 & $49 \%$ & $55 \%$ & $61 \%$ & $68 \%$ & $75 \%$ & $83 \%$ & $91 \%$ & $100 \%$ \\
\hline
\end{tabular}

$50 \sim 60 \%$ overall Viterbi constitency corresponds then to $65 \sim 75 \%$ individual success rate, which is optimistic.

2 According to the preceding table, this corresponds to a structural consistency of $95 \%$ for each component, which seems impossible to achieve by strictly automatic means in practical applications involving general users. 
because he sees it on his screen and steps in. In cases where users could not achieve satisfactory results by using (and helping) the system, the human expert would take charge of (part of) the translation.

We suppose an architecture flexible enough to allow the above three extralinguistic processes to be optional, and, in the case of interactive disambiguation, to allow users to control the amount of questions asked by the system. Hence, some ambiguities may remain after extralinguistic disambiguation. They should be solved by the system heuristically and "unsurely", by using preferences, scores or defaults. In that case, it is important that the questions asked from the users are the most crucial ones, so that failure of the last step to select the correct interpretation does not result in too damaging translation errors.

The questions we want to study on "ambiguity labelled" dialogues and texts are the following:

- what kinds of ambiguities (unsolvable by state-ofthe-art speech and text analyzers) are there in real data to be handled by the envisaged systems?

- what are the possible methods of interactive disambiguation, for each ambiguity type?

- how can a system determine whether it is important or not for the overall communication goal to disambiguate a given ambiguity?

- what kind of knowledge is necessary to solve a given ambiguity, or, in other words, whom should the system ask: the user, the interpreter, or the expert system, if any?

- in a given dialogue or document, how far do solutions to ambiguities carry over: to the end of the piece, to a limited distance, or not at all?

Ambiguity labelling should not be performed with reference to any particular analyzer, even if a good one is available. It should be done at a less specific level, suitable for generating disambiguation dialogues understandable by non-specialists. For example, attachment ambiguities are represented differently in the outputs of various analyzers, but it is always possible to recognize such an ambiguity, and to explain it by using a "skeleton" flat bracketing. Ambiguity labelling may also be considered as part of the specification of present and future state of the art analyzers, which means that:

- it should be compatible with the representation systems used by the actual or intended analyzers.

- it should be clear and simple enough for linguists to do the labelling in a reliable way and in a reasonable amount of time.

Finally, our labelling should only be concerned with the final result of analysis, not in any intermediate stage, because we want to retain only ambiguities which would remain unsolved after the complete automatic analysis process has been performed.

\section{Representations, Ambiguities and Associated Notions}

Even if we want to label ambiguities independently of any specific analyzer, we must have in mind a certain class of possible representation systems for analysis results, and to be clear about what an "ambi- guous representation" is and about what counts as an ambiguity, etc.

What is an "ambiguous representation"? This question is not as trivial as it seems, because it is often not clear what we exactly mean by "the" representation of an utterance. In the case of a classical context-free grammar $\mathrm{G}$, shall we say that $a$ representation of $U$ is any tree $T$ associated to $U$ via $G$, or that it is the set of all such trees? Usually, linguists say that $U$ has several representations with reference to $\mathrm{G}$.

But if we use f-structures with disjunctions, $U$ will always have one (or zero!) associated structure $S$. Then, we would like to say that $S$ is ambiguous if it contains at least one disjunction. Returning to $\mathrm{G}$, we might then say that "the" representation of $U$ is the disjunction of all trees $T$ associated to $U$ via $G$.

In practice, however, developers prefer to use hybrid data structures to represent utterances. Trees decorated with various types of structures are very popular. For speech and language processing, lattices bearing such trees are also used, which means at least 3 levels at which a representation may be ambiguous.

Which class of representation systems do we consider in our labelling? First, they must be fine-grained enough to allow the intended operations. For instance, text-to-speech requires less detail than translation. On the other hand, it is counter-productive to make too many distinctions. For example, what is the use of defining a system of 1000 semantic features if no system and no lexicographers may assign them to terms in an efficient and reliable way? Second, there is a matter of taste and consensus: although different representation systems may be formally equivalent, researchers and developers have their preferences. Third, the representations should be amenable to efficient computer processing. Let us make this point more precise.

A "computable" representation system is a representation system for which a "reasonable" parser can be developed.

A "reasonable" parser is a parser such as:

- its size and time complexity are tractable over the class of intended utterances;

- assumptions about its ultimate capabilities, especially about its disambiguation capabilities, are realistic given the state of the art.

A representation will be said to be ambiguous if it is multiple or underspecified.

In all known representation systems, it is possible to define "proper representations", extracted from the usual representations, and ambiguity-free. For example, if we represent "We read books" by the unique decorated dependency tree:

$$
\begin{aligned}
& \text { [ [ "We" ((1ex "I-Pro") (cat pronoun) } \\
& \text { (person 1) (number plur)...) } \\
& \text { "read" ((lex "read-V") (cat verb) } \\
& \text { (person 1) (number plur) } \\
& \text { (tense (pres past\})...) } \\
& \text { ["books" ((1ex "book-N") (cat noun)...)]] }
\end{aligned}
$$

there would be 2 proper representations, one with (tense pres), and the other with (tense past). 
For defining the proper representations of a representation system, it is necessary to specify which disjunctions are exclusive, and which are inclusive.

A representation in a formal representation system is proper if it contains no exclusive disjunction.

The set of proper representations associated to a representation $R$, is obtained by expanding all exclusive disjunctions of $R$ (and eliminating duplicates). It is denoted here by $\operatorname{Proper}(R)$.

$\mathrm{R}$ is multiple if IProper( $\mathrm{R}) \mathrm{l}>\mathrm{I}$. $\mathrm{R}$ is multiple if (and only if) it is not proper.

A proper representation $\mathrm{P}$ is underspecified if it is undefined with respect to some necessary information.

There are two cases: the information is specified, but its value is unknown, or it is missing altogether.

The first case often happens in the case of anaphoras: (ref ?), or in the case where some information has not been exactly computed, e.g. (task_domain ?), (decade_of month ?), but is necessary for translating in at least one of the target languages. It is quite natural to consider this as ambiguous. For example, an anaphoric reference should be said to be ambiguous

- if several possible referents appear in the representation (several proper representations),

- and also if the referent is simply marked as unknown, which causes no disjunction.

The second case may never occur in representations where all attributes are present in each decoration. But, in a standard $f$-structure, one cannot force the presence of an attribute, so that a necessary attribute may be missing: (ref ?) means the absence of attribute ref.

For any formal representation system, then, we must specify what the "necessary information" is. Contrary to what is needed for defining Proper( $R$ ), this may vary with the intended application.

Our final definition is now simple to state.

A representation $\mathrm{R}$ is ambiguous if it is multiple or if Proper(R) contains an underspecified $P$.

We distinguish three levels of granularity.

- a dialogue (resp. a text) can be segmented in at least two different ways into turns (resp. paragraphs), or

a turn (resp. a paragraph) can be segmented in at least two different ways into utterances. or

. an utterance can be analyzed in at least two different ways, whereby the analysis is pertormed in view of translation into one or several languages in the context of a certain generic task.

$\Lambda$ mbiguities of segmentation into paragraphs may occur in written texts, if, for example, there is a separation by a $<$ new_line> character only, without <line_feed $>$ or <paragraph $>$. They are much more frequent and problematic in dialogues. We found many examples of such ambiguities in ATR's transcriptions of Wizard of $\mathrm{Oz}$ interpretations dialogues [10].

Ambiguities of segmentation into utterances are frequent, and most annoying, as analyzers generally work utterance by utterance, cven if they can access analysis results of the preceding context. For example: "right
|? now |? turn left..." or ([10], p. 50): "OK |? so go back and is this number three |? right there |? shall I wait here for the bus?".

As far as utterance-level ambiguities are concerned, let us stress again that we consider only those which should be produced by a state-of-the-art analyzer constrained by the task. For instance, "Please state your phone number" should not be deemed ambiguous, as no complete analysis should allow "state" to be a noun, or "phone" to be a verb. That could be different in a context where "state" could be construed as a proper noun ("State"), for example in a dialogue involving the State Department.

There is a further point. Consider the utterance:

(1) Do you know where the international telephone services are located?

The underlined fragment has an ambiguity of attachment, because it has two different "skeleton" [2] representations:

[international telephone] services

/ international [telephone services]

As a title, this sequence presents the same ambiguity. However, it is not enough to consider it in isolation. Take for example:

(2) The international telephone services many countries.

The ambiguity has disappeared! It is indeed frequent that an ambiguity relative to a fragment appears, disappears and reappears as one broadens its context. For example, in

(3) The international telephone services many countries have established are very reliable.

the ambiguity has reappeared. Hence, in order to define properly what an ambiguity is, we must consider the fragment within an utterance, and clarify the idca that the fragment is the smallest (within the utterance) where the ambiguity can be observed.

Although utterance-level ambiguities must be considered in the context of whole utterances, a sequence like "international telephone services" is ambiguous in the same way in utterances (1) and (3) above. We call this an "ambiguity kernel", as opposed to "ambiguity occurrence", or "ambiguity" for short.

It also clear that another sequence, such as "important business addresses", presents the same sort of ambiguity, or "ambiguity type" in analogous contexts (here, "ambiguity of attachment", or "structural ambiguity"). Other types concern the acceptions (word senses), the functions (syntactic or semantic), ctc. "Ambiguity patterns" are more specific kinds of ambiguity types, usable to trigger actions, such as the production of disambiguating dialogues.

We take it for granted that, for each considered representation system, we know how to define, for each fragment $\mathrm{V}$ of an utterance $U$ having a proper representation $P$, the part of $P$ which represents $V$.

For example, given a context-free grammar and an associated tree structure $P$ for $U$, the part of $P$ representing a substring $V$ of $U$ is the smallest subtree $Q$ containing all leaves corresponding to $V . Q$ is 
not necessarily the whole subtree of $P$ rooted at the root of $Q$. Conversely, for each part $Q$ of $P$, we suppose that we know how to define the fragment $V$ of $U$ represented by $Q$.

Let $\mathbf{P}$ be a proper representation of $\mathrm{U} . \mathrm{Q}$ is a minimal underspecified part of $\mathrm{P}$ if it does not contain any strictly smaller underspecified part Q'

Let $P$ be a proper representation of $U$ and $Q$ be a minimal underspecified part of $P$. The scope of the ambiguity of underspecification exhibited by $Q$ is the fragment $V$ represented by $Q$.

In the case of an anaphoric element, $Q$ will presumably correspond to one word or term $\mathrm{V}$. In the case of an indeterminacy of semantic relation (deep case), e.g. on some argument of a predicate, Q would correspond to a whole phrase $\mathrm{V}$.

A fragment $V$ presents an ambiguity of multiplicity $n$ $(n \geq 2)$ in an utterance $U$ if it has $n$ different proper representations which are part of $n$ or more proper representations of $\mathrm{U}$.

$\mathrm{V}$ is an ambiguity scope of an ambiguity if it is minimal relative to that ambiguity. This means that any strictly smaller fragment $\mathrm{W}$ of $\mathrm{U}$ has strictly less than $\mathrm{n}$ associated sub-representations or, equivalently, that at least two of the representations of $\mathrm{V}$ are be equal with respect to $W$.

In example (1) above, then, the fragment "the international telephone services", together with the two skeleton representations

the [international telephone] services

/ the international [telephone services]

is not minimal, because it and its two representations can be reduced to the subfragment "international telephone services" and its two representations (which are minimal).

This leads us to consider that, in syntactic trees, the representation of a fragment is not necessarily a "horizontally complete" subtree. In the case above, for example, we might have the configurations given in the figure below.

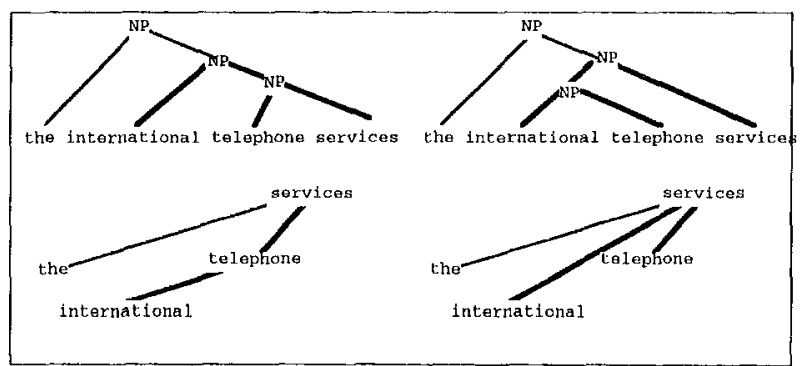

In the first pair (constituent structures), "international telephone services" is represented by a complete subtree. In the second pair (dependency structures), the representing subtrees are not complete subtrees of the whole tree.

An ambiguity occurrence, or simply ambiguity, A, of multiplicity $n(n \geq 2)$ relative to a representation system $R$, may be formally defined as:

$$
\mathrm{A}=\left(\mathrm{U}, \mathrm{V},<\mathrm{P}_{1}, \mathrm{P}_{2} \ldots \mathrm{P}_{\mathrm{m}}>,\left\langle\mathrm{p}_{1}, \mathrm{p}_{2} \ldots \mathrm{p}_{\mathrm{n}}>\right),\right.
$$

where $m \geq n$ and:
- $\mathrm{U}$ is a complete utterance, called the context of the ambiguity.

- V is a fragment of $U$, usually, but not necessarily connex, the scope of the ambiguity.

- $P_{1}, P_{2} \ldots P_{m}$ are all proper representations of $U$ in $R$, and $p_{1}, p_{2} \ldots p_{n}$ are the parts of them which represent $\mathrm{V}$.

- For any fragment.W of $U$ strictly contained in $\mathrm{V}$, if $\mathrm{q}_{1}, \mathrm{q}_{2} \ldots \mathrm{q}_{\mathrm{n}}$ are the parts of $\mathrm{p}_{1}, \mathrm{p}_{2} \ldots \mathrm{p}_{\mathrm{n}}$ corresponding to $\mathrm{W}$, there is at least one pair $\mathrm{q}_{\mathrm{i}}, \mathrm{q}_{\mathrm{j}}\left(\mathrm{i} \neq \mathrm{j}\right.$ ) such that $\mathrm{q}_{\mathrm{i}}=\mathrm{q}_{\mathrm{j}}$.

This may be illustrated by the following diagram,
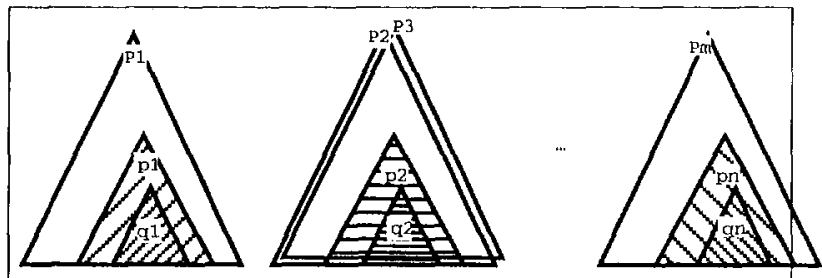

F

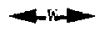

where we take the representations to be tree structures represented by triangles. Here, $\mathrm{P}_{2}$ and $\mathrm{P}_{3}$ have the same part $\mathrm{p}_{2}$ representing $\mathrm{V}$, so that $\mathrm{m}>\mathrm{n}$.

The kernel of an ambiguity

$A=\left(U, V,\left\langle P_{1}, P_{2} \ldots P_{m}\right\rangle,\left\langle p_{1}, p_{2} \ldots p_{n}\right\rangle\right)$ is the scope of $\mathrm{A}$ and its (proper) representations:

$\mathrm{K}(\mathrm{A})=\left(\mathrm{V},<\mathrm{p}_{1}, \mathrm{p}_{2} \ldots \mathrm{p}_{\mathrm{n}}>\right)$.

In a data base, it suffices to store only the kernels, and references to the kernels from the utterances.

The type of $\mathrm{A}$ is the way in which the $\mathrm{p}_{\mathrm{i}}$ differ, and must be defined relative to each particular $R$.

If the representations are complex, the difference between two representations is defined recursively. For example, two decorated trees may differ in their geometry or not. If not, at least two corresponding nodes must differ in their decorations.

Further refinements can be made only with respect to the intended interpretation of the representations. For example, anaphoric references and syntactic functions may be coded by the same kind of attribute-value pairs, but are usually considered as different ambiguity types.

When we define ambiguity types, the linguistic intuition should be the main factor to consider, because it is the basis for any disambiguation method.

For example, syntactic dependencies may be coded geometrically in one representation system, and with features in another, but disambiguating questions should be the same. Finally,

An ambiguity pattern is a schema with variables which can be instantiated to a (usually unbounded) set of ambiguity kernels.

Here is an ambiguity pattern of multiplicity 2 corres ponding to the example above (constituent structures).

$\mathrm{NP}\left[\mathrm{x} 1 \mathrm{NP}\left[\begin{array}{ll}\mathrm{x} 2 & \mathrm{x} 3]\end{array}\right], \operatorname{NP}[\mathrm{NP}[\mathrm{x1} \times \mathrm{x} 2] \times \mathrm{x} 3]\right.$.

We don't elaborate, as ambiguity patterns are specific to particular representation systems and analyzers, so that they should not appear in our labelling. 


\section{Principles of Ambiguity Labelling}

For lack of space, we cannot give here the contextfree grammar which defines our labelling formally, and illustrate the underlying principles by way of examples from a dialogue transcription taken from [1].

The labelling begins by listing the text or the transcription of the dialogue, thereby indicating segmentation problems with the mark " || ? ". Bracketed numbers are optional and correspond to the turns or paragraphs as presented in the original.

\section{LABELLED DIALOGUE: "EMMI 10a"}

[1] A: Good morning conference office $\|$ ? how can I help you

[2] AA: [ah] yes good morning could you tell me please how to get from Kyoto station to your conference center

[7] A: /Is/ OK, you're at Kyoto station right now $\|$ ?

[8] AA: \{yes\}

[9] A: $\{/$ breath/ $\}$ and to get to the International Conference Center you can either travel by taxi bus or subway how would you like to go

[10] AA: I think subway sounds like the best way to me

The labelling continues with the next level of granularity, paragraphs or turns. The difference is that a turn begins with a speaker's code. For each paragraph or turn, we then label the ambiguities of each possible utterance. If there is an ambiguity of segmentation in paragraphs or turns, there may be more labelled paragraphs or turns than in the source. For example, $A$ $\|$ ? $\mathrm{B} \|$ ? C may give rise to $\mathrm{A}-\mathrm{B} \| \mathrm{C}$ and $\mathrm{A} \| \mathrm{B}-\mathrm{C}$, and not to $\mathrm{A}-\mathrm{B}-\mathrm{C}$ and $\mathrm{AllB} \| \mathrm{C}$. Which combinations are possible should be determined by the person doing the labelling. An interruption such as [8] may also create a discontinuous turn $([7,9]$ here $)$.

In the case of utterances, the same remarks apply. However, discontinuities should not appear. There are often less possible utterances than all possible combinations. Take the example given in II. 3 above:

OK $\mid$ ? so go back and is this number three

|? right there ? shall I wait here for the bus?

This is an A I? B I? C I? D pattern, giving rise to 10 possible combinations. If the labeller considers only the 4 possibilities A|B|C-D, A|B|CID, A|B-CID, and A$\mathrm{B}-\mathrm{CID}$, the following 7 utterances will be labelled:

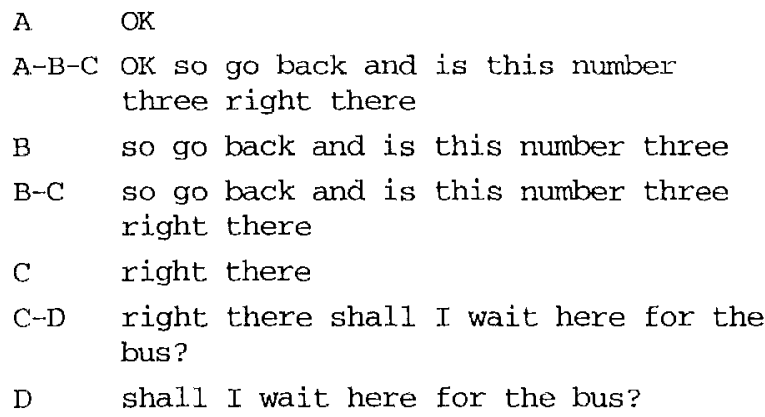

The mark TURN (or PARAG for a text) must be used if there is more than one utterance. /TURN is optional and should be inserted to close the list of utterances, that is if the next paragraph contains only one utterance and does not begin with PARAG. A format still closer to the TEI guidelines may be proposed in the future.

\section{LABELLED TURNS OF DIALOGUE "EMMI 10a" TURN}

[1] AA: Good morning, conference office, I? How can I help you?

UTTERANCES

[1.1] AA: Good morning, conference office (1)

(ambiguity EMMI10a-1-2.2.8.3 (

(scope "conference office")

(status expert_system)

(type address (*speaker *hearer))

(importance not-important)

(multimodal facial-expression)

(desambiguation_scope definitive)))

[1.2] AA: How can I help you?

... ambiguities

[1.1, 2] AA: Good morning, conference office, how can I help you?

... ambiguities

TURN

[2] AA: [ah] yes, good morning. I Could you tell me please how to get from Kyoto station to your conference center? ... ambiguities

\section{UTTERANCES}

The labeller indicates here a sure segmentation.

[2.1] AA: [ah] yes(2), good morning.

[2.2] AA: Could you tell me please how to get from Kyoto station to your conference center(3)?

The idea is to label all ambiguity occurrences, but only the ambiguity kernels not already labelled. The end of the scope of each ambiguity occurrence is indicated in the text by a bracketed number which identifies its ambiguity kernel.

Each ambiguity kernel begins with its header. Then come its obligatory labels (scope, then status, importance, and type, in any order), and its other labels. For example, the kernel header "ambiguity EMMI10a-2' 5.1 " identifies kernel \#2' in dialogue EMMI 10a, noted here EMMI10a. " 5.1" is the coding of [11].

The status (expert_system, interpreter, user) expresses the kind of supplementary knowledge needed to reliably solve the considered ambiguity. If "expert_system" is given, and if a disambiguation strategy decides to solve this ambiguity interactively, it may ask: the expert system, if any; the interpreter, if any; or the user (speaker). If "interpreter" is given, it means that an expert system of the generic task at hand could not be expected to solve the ambiguity.

The importance (crucial, important, not-important, negligible) expresses the impact of solving the ambiguity in the context of the intended task. Then comes 
the ambiguity type (structure, comm_act, class, meaning, target language, reference, address, situation, mode) and its value(s). The linguists may define more types and complete the list of values if necessary.

Other labels are optional. Their list will be completed in the future as more ambiguity labelling is performed. As for now, they comprise the disambiguation scope (how far does the solution of the ambiguity kernel carry over in the subsequent utterances), and the multimodality (what kind of cues could be used to help solve the ambiguity in a multimodal setting).

For lack of space, we can present only a few of the interesting examples from the same dialogue.

[4] AA: yes I am to(5) attend thi [uh]

Second International Symposium \{on\}

Interpreting Telecommunications

(ambiguity EMMI10a-5-3.1.2 (

(scope "am to")

(status user)

(type Japanese（"ねばならない"

$$
\text { "ことになっている" "はずだ")) }
$$

(importance important)))

The interpretation of "I am to" (obligation or future) is solvable reliably only by the speaker.

The following example is like the famous one: "Time flies like an arrow"! "Linguist's examples" are often derided, but they really appear in texts and dialogues.

However, as soon as they are taken out of context, they look again as artificial as "linguist's examples"!

[10] AA: I think subway sounds(10)

like(11) the best way to me

(ambiguity EMMI10a-10-3.1.1 (

(scope "sounds")

(status interpreter)

(type cat (verb noun))

(importance crucial)

(multimodal (prosody pause)))

(ambiguity EMMI10a-11-3.1.1 (

(scope "like")

(status interpreter)

(type cat (verb preposition))

(importance crucial)

(multimodal (prosody pause)))

Here is an example of communication-act ambiguity, which is crucial for translating into Japanese.

[11] A: OK, [ah] you wanna go by subway and you're at the station right now(12).

(ambiguity EMMI10a-12-5.1 (

(scope "you wanna go by subway and you're at the station right now")

(status expert-system)

(type CA (yn-question inform))

(importance crucial)

(multimodal prosody)))

\section{Conclusion}

Although many studies on ambiguities have been published, the specific goal of studying ambiguities in the context of interactive disambiguation in text and speech translation has led us to explore new ground and to propose the concept of "ambiguity labelling". About 80 pages of dialogues gathered at ATR have been labelled: monolingual dialogues in Japanese and English, and bilingual WOZ dialogues [10]. Attempts have also been made on French texts and dialogues, and on monolingual telephone dialogues for which analysis results produced by automatic analyzers were available. Part of these collected ambiguities have been used for experiments on interactive disambiguation.

\section{Acknowledgments}

Our thanks go to Dr. Y. Yamazaki, president of ATR-ITL, Mr. T. Morimoto, head of Department 4, and Dr. K.-H. Loken-Kim, for their constant support to this project, and to its funders, CNRS and ATR.

\section{References}

[1] ATR-ITL (1994) Transcriptions of English Oral Dialogues Collected by ATR-ITL using EMMI. TR-IT0029, ATR-ITL, January 1994, 33 p.

[2] Black E., Garside R. \& Leech G. (1993)

Statistically-Driven Grammars of English: the IBM/Lancaster Approach. J. Aarts \& W. Mejs, ed., Language and Computers: Studies in Practical Linguistics, Rodopi, Amsterdam, 248 p.

[3] Blanchon H. (1994) Perspectives of DBMT for monolingual authors on the basis of LIDIA-l, an implemented mockup. Proc. 15th International Conference on Computational Linguistics, COLING-94, Kyoto, Japan, 5-9 Aug. 1994, vol. 1/2, pp. 115-119. [4] Boitet C. (1993) Practical Speech Translation Systems will integrate human expertise, multimodal communication, and interactive disambiguation. Proc. MTS-IV, Kobe, 18-22 July 1993, pp. 173-176.

[5] Boitet C. \& Blanchon H. (1994)

Multilingual Dialogue-Based MT for Monolingual Authors: the LIDIA Project and a First Mockup. Machine Translation, 9/2, pp. 99-132.

[6] Boitet C. \& Loken-Kim K.-H. (1993)

Human-Machine-Human Interactions in Interpreting Telecommunications. Proc. International Symposium on Spoken Dialogue, Tokyo, 10-12 November 1993, Waseda University, $4 \mathrm{p}$.

[7] Maruyama H., Watanabe H. \& Ogino S. (1990) An Interactive Japanese Parser for Machine Translation. Proc. COLING-90, Helsinki, 20-25/8/90, II. Karlgren, ed., ACL, vol. 2/3, pp. 257-262.

[8] Park Y.-D. \& Loken-Kim K.-H. (1994) Text Database of the Telephone and Multimedia Multimodal Interpretation Experiment. Technical Report, ATR-ITL, Dec. $94,161 \mathrm{p}$.

[9] Park Y.-D., Loken-Kim K.-H. \& Fais I. (1994) An Experiment for telephone versus multimedia multimodal Interpretation: Methods and Subject's Behavior. Technical Report, ATR-ITL, Dec. 94, 15 p.

[10] Park Y.-D., Ioken-Kim K.-H., Mizunashi S. \& Fais L. (1995) Transcription of the Collected Dialogue in a Telephone and Multimedia/Multimodal WOZ Experiment. Technical Report, ATR-ITL, Feb. 95, 123 p. [11] Tomokiyo M. (1994) Ambiguity Classification and Representation. Proc. Natural Language Understanding and Models of Communication, Oct. 94 